\title{
INDICE DEI NOMI
}

Abbrugiati, Raymond, 270

Acocella, Nicola, 9, 196

Addison, Joseph, 264

Agnati, Achille, 474

Agnesi, Enrica, 259

Agostino, Santo, 432

Ailly, Marie-François d', 133

Ajello, Riccardo, 405, 438-439, 448

Albertone, Manuela, 7, 69

Albertoni, Ettore Adalberto, 297

Alembert, Jean-Baptiste Le Rond d', 413

Alessi, Giorgia, 401

Alighieri, Dante, 269

Alimento, Antonella, 86, 109, 113-116, 119-120, 123-124, 129-132, 182

Allegrini, Giuseppe, 98

Allen, Steward Lee, 262, 264

Ambrosoli, Luigi, 297, 399

Anderson, Benedict, 291

Antonielli, Livio, 401-402

Apih, Elio, 339, 395

Appiah, Kwame Anthony, 291

Archinti, Siro, 318-319

Arnaldi, Girolamo, 259

Asso, Pier Francesco, 121

Astigarraga, Jesus, 121

Audegean, Philippe, 26, 36, 92

Audibert, Pierre, 115

Augello, Massimo Mario, 473

Bacon, Francis, 147, 268, 403-404, 415, 428

Bagiotti, Anna, 448, 473

Bagiotti, Tullio, 448, 473

Baia Curioni, Stefano, 401

Baldus de Ubaldis, 121

Bandini, Sallustio, 204-205

Baranzini, Mauro, 30, 36

Barbarisi, Gennaro, 144, 151, 246-247, 260-261, 269, 286, 397-398, 400

Barbieri, Katherine, 286

Barbò, "ispettore", 332
Baron, Hans, 451

Bartesaghi, Paolo, 246

Bartolo da Sassoferrato, 121

Barucci, Piero, 37, 102, 379-380, 395, 399, 473

Baudeau, Nicolas, 80-83, 99, 103-105

Baumol, William J., 62-63

Bealer, Bonnie K., 263

Beaumont vedi: Moreau de Beaumont, Jean-Louis

Becagli, Vieri, 102

Beccaria, Cesare, 4, 7, 9-10, 13-16, 18-19, 23-41, 43, 70-71, 84-85, 87, 89-94, 96$101,104-105,108,120,137-149,152-$ $162,165-170,173,184-185,187,192-$ 196, 198-200, 202, 208-210, 214, 216227, 229-240, 244-246, 248, 251, 254259, 261, 274, 293, 295-312, 316-336, $338-341,343-357,359-366,368-379$, 381-404, 406-407, 409, 416-417, 421, $425-426,428,431-433,438-440,448$, 460, 466-467, 470-471, 473

Becker, Gary, 62

Bellanca, Nicolò, 451, 456, 473

Belloni, Girolamo, 214

Bentham, Jeremy, 440-441

Benton, Lauren, 289

Benucci, Antonio, 103

Berg, Maxine, 69

Berlinguer, Luigi, 397, 401

Berns, Thomas, 123

Bertier de Sauvigny, Louis-Jean, 132

Bertin, Henri, 116, 119

Besozzi, "vice visitatore generale", 300, 315-316

Bianchini, Marco, 184, 219, 406

Bielfeld, Jacob Friedrich von, 93

Biffi, Giovan Battista, 247, 249, 258

Bigatti, Francesco, 111-112

Bigot de Sainte Croix, Louis-Claude, 9899 
Biscardi, Luigi, 401

Bitterling, David, 113

Blanvillain, Jean-François, 103

Blasco, Teresa, 417

Blaug, Mark, 63

Bleek, Wilhelm, 261

Bloch, Marc, 236

Boccapaduli Sparapani Gentili, Margherita, marchesa, 247

Bocer, Heinrich, 121-122

Bodin, Jean, 123

Bognetti, Giuseppe, 7, 33, 36-37, 84, 137, 149, 177, 180-181, 184-185, 346, 408, 412, 473

Bonaparte, Napoleone, 49-50

Bonnafous, Alain, 51

Borioli, Daniele, 114-115

Borruso, Edoardo, 400

Bortolotti, Mariapia, 36, 401

Botero, Giovanni, 43

Bourillon, Florence, 135

Bousquet, Georges-Henri, 237

Bouvier, Michel, 114, 123

Bracciolini, Poggio, 434, 451

Brambilla, Elena Cristina, 357, 395, 401

Braudel, Fernand, 236

Breen, Timothy H., 276

Bresciani-Turroni, Costantino, 239-240

Breton, Yves, 50-51, 74, 76

Broggia, Carlo Antonio, 124, 214

Brown, Howard G., 69

Brucker, Gene A., 288

Bruni, Leonardo, 451

Bruni, Luigino, 7, 90, 431, 433, 438-439, 441, 445, 448, 456, 458, 466, 471, 473

Burckhardt, Jacob, 451

Burgio, Alberto, 359, 395

Burke, Peter, 265

Burrows, Simon, 91

Butel-Dumont, Georges-Marie, 82, 274

Butterfield, Herbert, 20, 36

Cafagna, Luciano, 401

Caffè, Federico, 196, 238-240

Caizzi, Bruno, 303, 308, 340-342, 395
Campioni, costruttori di "impianti per la raffinazione del ferro", 309

Canetta, Rosalba, 298, 300-301, 304, 307, $311-312,317,322,344,348,388,390-$ 392, 395-396, 400-401

Cannan, Edwin, 454-455

Canosa, Romano, 401

Cantarutti, Giulia, 260

Cantillon, Richard, 40-41, 78, 99-101, 411

Cantù, Cesare, 399

Capelli, Anna, 401

Capra, Carlo, 3, 7, 9, 36-37, 86-87, 93, $112-113,115,120-121,141,144,150-$ 151, 168, 173-174, 184-185, 189, 191, 243, 247, 257, 259-261, 267-268, 271, $293,303,308,355,357,359,370,373$ $374,379,383,386,388,391,395,400$ 402, 406, 411, 417, 457, 473

Caracciolo, Albert, 206, 215

Carera, Aldo, 173, 184, 374, 378, 390, 393, 396, 401

Carli, Gian Rinaldo, 108, 110, 114, 119 120, 190, 214, 219, 224, 226, 243-244, 251-252, 258-259, 266, 274, 278, 285, 287-289, 297, 304, 338-339, 373-374, 387, 390, 406, 408

Carli, Girolamo, 369

Carlo Magno, imperatore, 199

Carlo V d'Asburgo, imperatore, 110-111, 253

Carlo VI d'Asburgo, imperatore, 113, 121, 151, 249, 276, 279

Carmichael, Gershom, 458

Carmignani, Giovanni, 389, 396

Carnino, Cecilia, 7, 69

Carozzi, Carlo, 115

Carpanetto, Dino, 260, 286, 460, 473

Carpani, Francesco Maria, 178-179

Cartelier, Jean, 49

Carter, April, 290

Cary, John, 262-263, 274

Casanova, Giacomo, 264

Casati, Carlo, 102, 271 
Casini, Paolo, 413

Cassi, Aldo A., 357, 396

Castel de Saint-Pierre, Charles-Irénée, 122-123

Castelli, Giovanni, 124

Castronovo, Valerio, 100

Catalano, Franco, 399

Cattaneo, Carlo, 244, 296-297, 398

Cattaneo, Mario Alessandro, 299, 361, 396, 400

Cattini, Marco, 278

Cavanna, Adriano, 312, 390, 396, 400

Celesia, Pietro Paolo, 408

Cereghini, Bernadette, 36, 401

Ceva, Giovanni, 405-406

Charles, Loic, 73, 77

Chauvelin, François-Claude, marchese di, 117

Chiappetti, Achille, 371, 396

Chiari, Alberto, 321

Chiozza, Lorenzo, 127

Ciserani, Maria Teresa, 401

Clément, Alain, 80

Clemente XIV, 252

Cleopatra, 75

Clicquot de Blervache, Simon, 75

Clifford, Helen, 69

Cochrane, Eric, 258-259

Colao, Floriana, 397, 401

Colbert, Jean-Baptiste, 76, 99, 279

Colloredo, Girolamo, 175

Colonnello, Isabella, 401

Columella, Lucius Junius Moderatus, 419

Comba, Rinaldo, 112

Comisso, Giovanni, 264

Comparet, Jean-Antoine, 98

Condillac, Étienne Bonnot de, 40, 48, 89, 92, 268, 415

Condorcet, Marie Jean Antoine Caritat, marchese di, 13-14, 18, 266

Constancio, Francisco, 55

Contini, Alessandra, 401

Cooper, Anthony Ashley, conte di Shaftesbury, 72, 458
Copernico, Nicolò, 406

Corpaci, Francesco, 399

Cossa, Luigi, 399

Costabile, Lilia, 37

Costamagna, Henri, 116

Cova, Alberto, 406

Cowan, Brian, 263

Coyer, Gabriel-François, 73, 79

Cremani, Luigi, 386

Crepax, Nicola, 401

Cristiani, Beltrame, 119, 175, 178, 249 250

Croce, Benedetto, 144, 439, 448, 456

Crowely, John E., 88

Cuccia, Silvia, 369, 396

Cuoco, Vincenzo, 379, 397

Custodi, Pietro, 85, 108, 141, 199, 201204, 207-209, 214, 218, 221, 246, 463 465

D’Arco, Giambattista Gherardo, 103, 381

Dal Pane, Luigi, 399

Darby, John, 274

Davanzati, Bernardo, 201-202

David, Elisabeth, 62

De Amicis, Edmondo, 446

De Felice, Renzo, 92, 211, 461, 474

De Francesco, Antonino, 401

De Lorenzo, Renata, 397

Del Mercato, Barbara, 255

De Maddalena, Aldo, 151, 260-261, 269, 278, 286, 397-398, 400

De Mairville, conte, 135

De Marchi, Neil, 90

Descartes, René, 425

De Sauvigny, Bertier, 132

De Stefano, Francesco, 120, 259, 261, 266, 274, 278, 285, 288

De Tiberiis, Giuseppe, 438, 448

De Vries, Jan, 282

del Vecchio, Gustavo, 236-239

Della Silva, Paolo, 316

Demetrio, proprietario di caffe', 265 267, 287, 292, 417 
Dezza, Ettore, 400-401

Di Donato, Francesco, 147

Di Matteo, Massimo, 37

Di Renzo Villata, Maria Gigliola, 122, 362, 396

Di Simone, Maria Rosa, 351, 396

Diaz, Furio, 450, 473

Diderot, Denis, 248, 439

Dionisotti, Carlo, 288

Dipper, Christof, 151

Domat, Jean, 123

Donadeo, Giovanni Battista, 330-333

Donadeo, Michele, 311, 316-318, 320$321,326,330-331,385$

Donati, Claudio, 86-87

Doria, Paolo Mattia, 451

Dragonetti, Alfonso, 439, 448

Dragonetti, Giacinto, 7, 431-433, 438443, 448

Drèze, Jean, 33

Du Pont de Nemours, Pierre Samuel, 81, 97-99, 134, 476

Dubois, Auguste, 80

Dubourg-Glatigny, Pascal, 129

Dupac de Bellegarde, Gabriel-Marie, 99

Dupont, Jean-Claude K., 123-124

Duport, rappresentante all'Assemblée Nationale, 147

Dutot, Nicolas, 102

Dziembowski, Edmond, 91

Eger, Elizabeth, 69

Einaudi, Luigi, 144, 178, 180, 194-195, 198-199, 222-224, 227, 232-236, 399, 407

Ellis, Aytoun, 263

Ellis, Markman, 258, 262, 264

Enrico IV, re di Francia, 74

Errera, Alberto, 387, 390, 396

Eugenio, principe di Savoia-Carignano, 111, 113

Fabrini, Giovannantonio, 213

Faccini, Luigi, 400

Faucci, Riccardo, 89, 451, 473

Febvre, Lucien, 236
Federico II di Hohenzollern, re di Prussia, 257, 261

Ferdinando d'Asburgo-Lorena, arciduca d'Austria, 253, 322

Ferrara, Francesco, 170, 237, 455

Ferrari, Desiderio, 322

Ferrari, Luigi, 265

Ferrari, Stefano, 260, 322

Ferraris, Magda, 114

Ferriù, Pietro, 307

Ferrone, Vincenzo, 87, 395-396, 405

Ferroni, Pietro, 408

Filangieri, Gaetano, 170, 440-441, 466

Filippi, Paola Maria, 260

Firmian, Carlo Gottardo, 108, 115, 119 120, 128-129, 132, 249, 251-253, 285 , $304,312-313,315-316,352,392$

Firpo, Luigi, 85, 298, 300-301, 304, 307, $311-312,317,348,388,395-396,473$

Flaim, Carmen, 260

Fleury, Gabriel, 125

Fontenelle, Bernard de, 118

Forbonnais, François Véron Duverger de, 71, 73-76, 81-83, 91, 93, 96-97, 101-102, 105, 119, 125, 132, 271-273

Forti, Francesco, 389

Forti Messina, Annalucia, 400

Foscari, Giuseppe, 401

Foulquié, Paul, 58

Franci, Carlo Sebastiano, 83, 255-256, $258,261,269,280-285,289,293,418$ 419

Francioni, Gianni, 83, 85, 87, 142, 147, 184, 257, 281, 291, 298-301, 307, 311$312,317,348,391,395-396,415,425$, 473

Frank, Johann Peter, 311

Frey, Bruno S., 442, 448

Frisi, Paolo, 247, 251, 258, 403-404, 407408, 412-414, 428

Frumento, Armando, 305-306, 355, 396, 399

Fuensaldaña, Alfonso Perez de Vivero y Menchaca, conte di, 111

Gaeta, Giuseppe, 322-324 
Galasso, Giuseppe, 395, 405

Galbiati, Giovanni, 399

Galeazzi, Giuseppe, 98, 293

Galiani, Ferdinando, 13-14, 39-41, 84, 86, 206-207, 214-216, 218, 237, 244$245,247,405,408-409,432-433,473$

Galilei, Galileo, 268, 288, 403-404, 413 415, 425, 428

Gallarati Scotti, Francesco, 341

Galli, Giancarlo, 112, 271

Gallois, Léonard, 109

Gambi, Lucio, 115

Garat, rappresentante all'Assemblée Nationale, 147

Garin, Eugenio, 451-452, 474

Garlati, Loredana, 358, 369, 396

Garms Cornides, Elisabeth, 261

Garnier, Germain, 49, 117

Garofalo, Giuseppe, 471

Gaspari, Gianmarco, 298-299

Gattoni, imprenditore, 303-304, 309-310

Gaudin, Martin-Michel-Charles, duca di Gaeta, 133, 135

Gayer, Arthur D., 236

Gazzola, Giovanni Maria, 319-321

Genovesi, Antonio, 8, 13-14, 18, 84, 86, 99, 170, 173, 207-209, 244-245, 273, 432-434, 438-441, 443, 448, 452, 455, 458-459, 465, 470, 473-474

Geri, Marco Paolo, 389, 396

Ghiringhelli, Robertino, 297

Ghisalberti, Fausto, 321

Gianformaggio, Letizia, 89

Gibbs, Graham C., 260

Gioia, Melchiorre, 296, 338, 348, 373 375, 378-381, 398, 431-432, 441, 448

Giovio, Giambattista, 343

Giulini, Alessandro, 101, 120, 247

Giuntini, Vincenzo, 217

Giuseppe II d'Asburgo-Lorena, imperatore, 129-130, 132, 134, 145-146, 248, 253, 313, 356-358, 389, 397, 460, 462

Giussani, Ferrante, 309

Giusti, Luigi, 250
Gorla, Gino, 310, 397

Goudar, Ange, 264

Gournay, Vincent de vedi: Vincent de Gournay, Jacques Claude Marie

Grab, Alexander, 377, 382-383, 397, 400

Graber, Frédéric, 111

Grafton, Anthony, 292

Granito, Eugenia, 401

Grasset, François, 98

Gravier, Jean, 438-439

Gravisi, Girolamo, 339

Graziadei, Antonio, 456

Graziani, Augusto, 471

Greenfeld, Liah, 290

Greppi, Emanuele, 101, 120, 181, 189, 247, 279

Grinevald, Paul-Marie, 135

Groenewegen, Peter, 6, 9, 92, 95, 210211, 274

Guaita, imprenditori, 302-303

Guerci, Luciano, 473

Guerrieri, “delegato assessore”, 322

Guéry, Alain, 123

Guggenheim, Thomas, 41

Gui, Benedetto, 471

Guidi, Marco Enrico Luigi, 89, 473

Guilhamon, Henri, 133

Gutiérrez, Juan, 121

Habermas, Jürgen, 263, 270

Halevi, Joseph, 211

Harvey, William, 403-404, 428

Harvouin, François-Joseph, 109, 114, 116-117, 123, 128-130, 133, 135

Hashimoto, Hitoshi, 51-53

Hattox, Ralph S., 262

Hauc, Jean-Claude, 264

Hauteville, Joseph François Perret, conte di, 115

Hegel, Georg Wilhelm Friedrich, 266

Helvétius, Claude-Adrien, 96-97, 142, 435, 467

Hennet, Albert-Joseph-Ulpien, 133, 135

Hicks, John, 193-194, 196-197, 234

Hilaire-Perez, Liliane, 114 
Hirschman, Albert, 72, 286

Hobbes, Thomas, 25, 125, 432, 453, 459, 466

Holbach, Paul Henri Dietrich, barone d', 467

Hont, Istvan, 6, 9, 261, 474

Hopkins, Johns, 262, 264

Horodowich, Elizabeth, 263

Hotta, Seizo, 6, 9, 93, 271, 274, 409

Hsia, R. Po-chia, 265

Hufteau, Yves-Louis, 147

Hume, David, 71-72, 74-75, 83, 91, 102, $185,409,411-412,435,461,466$

Hutcheson, Francis, 72, 89, 142, 458

Hutchison, Terence, 140

Ignatieff, Michael, 6, 9, 474

Imbonati, Carlo, 245

Imbonati, Giuseppe Maria, 245

Imbruglia, Girolamo, 409

Intieri, Bartolomeo, 439, 458

Israel, Jonathan, 457, 467-468, 474

Jacobs, Helmut C., 270

Jonard, Norbert, 260, 286

Jossa, Bruno, 274, 474

Kant, Immanuel, 290

Kaunitz-Rittberg, Wenzel Anton von, $157,173,175-177,179,182,187,191$, 243-244, 249-252, 260, 268, 279, 285, $301,308,312-316,358,383,392$

Keynes, John Maynard, 197

King, Charles, 273-274

Klein, Lawrence E., 72

Klingenstein, Grete, 260

Klock, Caspar, 121-122

Kohn, Hans, 288

Krynen, Jacques, 122

Kwass, Michael, 78

La Mettrie, Julien Offray de, 467

Laband, Anton, 139, 147

Labriola, Antonio, 456

Labriola, Arturo, 456

Lambertenghi, Luigi, 258

Landais, Benjamin, 132

Landi, Sandro, 270

Lane, Frederic C., 236, 262
Lang, Peter, 27

Lange, Oskar, 62

Lapidus, André, 48, 59

Larrère, Catherine, 123, 125

Lather, Hermann, 121-122

Le Mercier de La Rivière, Pierre-PaulFrançois-Joachim-Henri, 100

Le Trosne, Guillaume-François, 126-127, 134

Lebeau, Christine, 7, 107, 116

Lefebvre, Frédéric, 73

Lefèvre d'Ormesson, Marie-François-dePaule, 130, 132-133

Legnani, amministratore, 339

Leopoldo II d'Asburgo-Lorena, imperatore, $150,300,310,312,341,349$, $370,386,389,460$

Levati, Stefano, 86

Lipsey, Richard G., 183

Liva, Giovanni, 112, 314, 316, 397

Livingstone, David, 443

Livorsi, Franco, 297

Lloyd, Henry, 271

Locke, John, 89, 138, 143-144, 146-147, 151, 409, 426, 461-462

Longo, Alfonso, 88-89, 159, 247-248, 251, 258, 268

Loria, Achille, 456

Lottinger, Stefano, 191

Luftalla, Michel, 50-51

Luigi XII, re di Francia, 259

Luigi XIV, re di Francia, 279

Lupi, Carlo, 135

Lutero, Martino (Martin Luther), 432

Luzzatto, Fabio, 399

Macchioro, Aurelio, 401

Machiavelli, Niccolò, 435-436, 452

Mahieu, François-Régis, 55

Maifreda, Germano, 7, 403, 406

Malherbe, Michel, 91

Mandeville, Bernard, 71, 75, 152, 435, 438, 459, 466

Mangio, Carlo, 401

Manini, Lorenzo, 103

Manini, Silvio, 332 
Mansfield, Edward D., 286

Manuzzi, Giovan Battista, 264

Manzoni, Alessandro, 245, 321

Manzoni, Massimiliano, 306

Marangoni, Giandemetrio, 474

Maraviglia Mantegazza, Angelo Maria, 171-172, 176, 180

Maria Teresa d'Asburgo, imperatrice, $117,151,245,249,253,277,460$

Marinoni, Giovanni Jacopo de, 112

Marjanen, Jani, 255

Martin, Thierry, 112

Martini, Carlo Antonio, 357-358, 396

Marx, Karl, 56, 454-456

Massetto, Giampaolo, 7, 295, 349, 361, 397, 401

Mata, Maria Eugénia, 9

Mauri, Angelo, 100, 399

Mazucchelli, Giovanna, 112

Mazzocco, Angelo, 288

Mellerio, Giacomo, 181, 185-187

Melon, Jean François, 71, 74-75, 83, 85, 93, 97, 101-102, 125, 273

Mercier, Ch., 47

Mercier de la Rivière, Pierre-PaulFrançois-Joachim-Henri vedi: Le Mercier de La Rivière, Pierre-PaulFrançois-Joachim-Henri

Merola, Alberto, 206, 215

Messbarger, Rebecca, 269-270

Michel, George, 43

Miglio, Gianfranco, 395

Mill, James, 63

Miller, Judith A., 69, 292

Miller, Peter N., 292

Mirabeau, Victor Riqueti, marchese di, 77-80, 88, 93, 99, 102-105, 247

Moioli, Angelo, 7, 22-23, 36-37, 84, 94, $171,174,177-178,180-181,183,185$,

187, 190-191, 346, 400, 408, 412, 473

Molla Losito, Valeria, 400

Mondolfo, Rodolfo, 36

Monfardini, imprenditore, 307

Monsaingeon, Guillaume, 112

Montanari, Geminiano, 202, 204, 406
Montanari, Giovanni, 438

Montesano, Aldo, 474

Montesquieu, Charles-Louis de Secondat, baron de, 71-72, 74-75, 83, 93, 96-97, $125,138,144,146-147,161,244-245$, 268, 272, 286, 290, 439, 461-464, 466

Montorzi, Mario, 396

Moreau de Beaumont, Jean-Louis, 117 . 118, 134-135

Morellet, André, 13-14, 18, 74, 97, 119, 131

Mornico, Francesco, 306-307, 355

Morosini, "magistrato politico camerale", 311

Morozzo, Carlo Filippo Vittorio, conte di Magliano, 117, 129

Mossner, Ernest C., 117

Mouchot, Claude, 48

Mozzarelli, Cesare, 124, 318, 340, 357, 381, 389, 395, 397, 400

Muratori, Lodovico Antonio, 85, 87, 214, 243-244, 251, 409, 452

Musa, Mark, 288

Muthu, Sankar, 290

Muttoni, Filippo, 178-179

Natoli, Salvatore, 459, 474

Negri Zamagni, Vera, 184, 474

Neppi Modona, Guido, 298, 350, 397

Neri, Pompeo, 7, 16, 85, 88, 107-108, 110, 113-114, 117, 119-120, 122-123, 127-132, 134, 201-202, 214-215, 224, 244, 250, 264, 297, 387

Newton, Isaac, 268, 403-404, 407, 413, $415,425,428$

Nicolas, Jean, 115

Novati, Francesco, 101, 247

Nutini, Stefano, 379, 397

Odelli, Lorenzo, 322

Olivi, Pietro, 433

Olmi, Giuseppe, 395

Oresko, Robert, 260

Ormesson vedi: Lefèvre d'Ormesson

Ottoboni, famiglia, 386

Outram, Dorinda, 263 
Padoa Schioppa, Antonio, 3-4

Pagano, Emanuele, 357, 397, 440

Pagnini, Giovanni Francesco, 214, 244

Paine, Thomas, 13-14, 18, 441

Pallares-Burke, Maria Lúcia, 265

Pallavicini, Gian Luca, 119, 243-244, 249-250

Palmieri, Matteo, 451

Panciera, Walter, 278

Panizza, Giorgio, 473

Pansini, Guseppe, 310, 397

Panzeri, Laura, 400

Paoli, Pasquale, 460

Paolo di Castro, 121

Pareto, Vilfredo, 456

Parini, Giuseppe, 245-246, 251

Parisi Acquaviva, Daniela, 271, 401

Parravicini, Carlo Francesco, 315

Pasinetti, Luigi L., 30, 36, 468-472

Passerini, Vincenzo, 443

Pasta, Renato, 87, 260, 386, 397

Patalano, Rosario, 274, 473-474

Pecchio, Giuseppe, 109, 399

Pecci, Nicola, 297

Peiresc, Nicolas-Claude Fabri de, 292

Pellanda, Anna, 474

Pellegrini, Antonio, 128, 132, 179, 181, 187

Pellegrini, Giuseppe, 340

Pellizzari, Fausta, 30, 37

Perego, Antonio, 258

Perna, Maria Luisa, 84, 448, 473

Perrot, Jean-Claude, 77

Perrotta, Cosimo, 177

Pertusati, "soprintendente alle fabbriche camerali", 312

Pestarino, Rossano, 346, 379

Petitti di Roreto, Giuseppe Antonio, 115

Petrarca, Francesco, 287-288

Petty, William, 125, 403-404, 410, 428, 454

Piccinini, "capo dell'ufficio di revisione della Camera dei Conti”, 191

Piermarini, Giuseppe, 311, 314
Pini, Ermenegildo, 305-307, 355

Pisani, Mario, 281, 321, 350, 397, 401402

Pisapia, Gian Domenico, 9, 92, 395-396

Pizzala, Antonio Maria, 335-336

Platone, 426

Plumard de Dangeul, Louis-Joseph, 71, 75, 85

Pocock, John Greville Agard, 272, 452, 467

Pollins, Brian M., 286

Pompadour, Jeanne-Antoinette, marchesa di, 73

Pope, Alexander, 264

Porret, Michel, 396-398

Porta, Pier Luigi, 4-6, 8-9, 21-22, 29, 33, $36-37,84,90,92,95,144,153,165$, $168,173,176-177,180,182,184,202$, $257,346-347,379,397,408-409,411$ 413, 449, 456, 461, 470-471, 473-474

Poullin de Viéville, Nicolas-Juste, 118

Pras Martiniana, conte di, 111-112, 121

Praz, Mario, 265

Premoli, Antonio, 114

Prony, Gaspard-Clair-François-Marie Riche de, 135

Provin, Gabriele, 357-358, 398

Psalidopoulos, Michalis, 9

Quadrio Curzio, Alberto, 4, 6-7, 9, 19, 30, 36-37, 90, 168, 193-197, 199, 210, 297, 398, 401, 471-472, 474

Quataert, Donald, 263

Quazza, Guido, 116

Quesnay, François, 13-14, 18, 40, 44, 46$47,49,71,73,77,79-81,97-99,101$ 103,453

Radicati di Cocconato, Ignazio, 408

Raviola, Blythe Alice, 112

Raynal, Guillaume-Thomas-François, 135

Razdolski, Roman, 134

Recalcati, Carlo Maria, 313

Reda, Giacomo, 328-329

Redaelli, "ispettore", 325 
Reill, Dominique Kirchner, 289

Reinert, Erik S., 281

Reinert, Sophus A., 7, 255, 271, 274, 281283, 290, 293, 474

Rétat, Pierre, 81

Rey, Guido M., 9, 196

Ricardo, David, 55, 453-455, 466

Ricca-Salerno, Giuseppe, 124

Ricci, Isabella, 115

Ricci, Lodovico, 387

Richeprey, Jean-François Henri de, 126, 133

Ricuperati, Giuseppe, 100, 260, 286, 460, 473

Risi, Paolo, 314, 341

Robertson, John, 270, 459, 474

Romagnoli, Sergio, 9, 36, 83, 85, 92, 147 $148,184,257,266,298,311,371,395$ $396,415,425,473$

Romagnosi, Giandomenico, 8, 10, 244

Romani, Mario, 295-296, 308, 385, 395, 398-400

Romani, Marzio Achille, 278

Romani, Roberto, 379, 398, 401

Roncaglia, Alessandro, 177

Roscher, Wilhelm, 122

Rosenberg, Nathan, 114

Ross, Ian S., 117

Rotelli, Ettore, 151, 260-261, 269, 286, 397-398, 400

Rothschild, Emma, 6, 10, 266

Rotondi, Claudia, 471

Rousseau, Jean-Jacques, 44, 98, 142, 244 $245,248,258,439,448$

Rovani, Giuseppe, 386-388

Rozdolski, Roman, 134

Ruffolo, Giorgio, 282

Rutto, Giuseppe, 286

Sacchi Landriani, Giannantonio, 3

Saint-André, Giuseppe, 339

Saint-Jean, Raymond, 58

Saint-Lambert, Jean-François de, 76, 9192

Saint-Pierre abbé vedi: Castel de SaintPierre, Charles-Irénée
Salvemini, Gaetano, 401

Santato, Guido, 86

Santucci, Antonio, 141, 316, 395

Say, Jean-Baptiste, 7, 39-44, 48-67, 81

Sbriccoli, Mario, 360, 398

Scaruffi, Gasparo, 406

Scazzieri, Roberto, 4-7, 9, 13, 19, 30, 3637, 95, 144, 168, 193, 196, 199, 210, 257, 379, 397, 401, 450, 461, 471, 474

Scazzoso, Mario, 400

Schabas, Margaret, 90

Schaub, Jean-Frédéric, 121

Schettini, Mario, 417

Schiavino, Mariateresa, 401

Schiera, Pierangelo, 400

Schlüter, Gisela, 270

Schumpeter, Joseph A., 40-42, 62-63, $140,170-172,180,182,210,220,255$ 256

Schumpeter, Elizabeth Boody, 210

Schwennicke, Andreas, 122

Scorza, Baldassarre, 172, 189-192, 353

Scott, Hamish M., 260

Scotti, Aurora, 400

Scotti Morgana, Silvia, 269

Secco Comneno, Pier Francesco, 185, 187,314

Sella, Domenico, 189, 259, 395

Sen, Amartya K., 9, 33, 37, 196

Seregni, Giovanni, 247

Sereno, Paola, 112

Serra, Antonio, 43, 202-203, 474

Severin, Dante, 399

Shackleton, Robert, 291

Shaftesbury (Cooper Anthony Ashley) vedi: Cooper, Anthony Ashley, conte di Shaftesbury

Shovlin, John, 69, 73, 269, 279

Silhouette, Etienne, 73, 135

Silvestri, Paolo, 474

Simili, Raffaella, 37

Sinzendorf, Philipp Ludwig Wenzel von, 255-256, 276-277, 279, 289

Skinner, Quentin, 272

Smith, Adam, 8, 39-40, 42-44, 49, 51-55, 
$63,66,81,92,117,139-140,153,161$ 162, 203-204, 220, 432-433, 435-438, 442 , 448, 453-456, 458-459, 462, 466467, 470, 473-474

Smith, Woodruff D., 264

Sofia, Francesca, 401

Sommaria, Matteo, 318

Sommi Picenardi, Guido, 249

Sonenscher, Michael, 261

Sori, Ercole, 400

Sormani, Giovanni Angelo, 300-301

Sowell, Thomas, 63

Spang, Rebecca L., 69

Spector, Céline, 72

Speelman, Patrick, 271

Spengler, Joseph J., 63

Sperges, Joseph, 250

Sraffa, Piero, 454, 456, 469

Stallybass, Peter, 263

Stapelbroek, Koen, 255, 458

Steele, Richard, 264

Stefani, Gaetano, 310

Steiner, Philippe, 51

Stendhal (Henry Beyle), 254

Stolleis, Michael, 122

Sugden, Robert, 433, 448, 458, 466, 471

Sully, Maximilien de Béthune, duca di, 108

Sundaram, Jomo K., 281

Swift, Jonathan, 264, 422

Szabo, Franz A.J., 187, 260

Taccolini, Mario, 401

Talamona, Mario, 297, 471

Tanucci, Bernardo, 13-14, 18

Tapinos, Georges, 49

Tarbé, Charles, 133

Tarello, Giovanni, 142

Taverna, "assessore provinciale della Giunta pie fondazioni”, 314

Théré, Christine, 73, 77

Thiessen, Hillard von, 116

Thomson, Ann, 91

Thweatt, William O., 63

Tiberi, Mario, 9, 196

Tiran, André, 7, 39, 62
Todeschini, Giacomo, 474

Tonelli, Giovanna, 36-37, 84, 177, 182, 184, 188-189, 346, 379, 408, 412, 473

Tooke, Thomas, 53-54

Torre, Angelo, 116, 184

Tortarolo, Edoardo, 270

Touzery, Mireille, 116

Trampus, Antonio, 287

Trask, Willard R., 264

Trezzi, Luigi, 302, 348, 398

Trotti, Giovanni Battista, 313

Trudaine de Montigny, Jean-CharlesPhilibert, 108

Tubaro, Paola, 405, 407, 409

Turgot, Anne-Robert-Jacques, barone de l'Aulne, 39-42, 48, 59, 81, 92, 117, 132-133, 467-468

Uztáriz, Jerónimo de, 108

Valeri, Nino, 460, 474

Valsecchi, Franco, 110, 387, 398

Vassalli, Giuliano, 350, 398

Vauban, Sébastien Le Prestre, marchese di, 112-113, 118, 121-123

Venn, John, 291

Venturi, Franco, 6, 10, 16, 26, 37, 40, 87, $108,114,214,243,245,248,257,259$, 261, 270-271, 387, 408-409, 457, 473 . 474

Verga, Ettore, 399

Vérin, Hélène, 111-112, 129

Verna, Catherine, 114

Verri, Alessandro, 101-102, 104-105, $120,141,146,150,166,245,248,253$. $254,258,268-271,279,403-404,417$ 419, 427-428

Verri, Gabriele, 121, 125, 134, 353

Verri, Pietro, 3, 7, 9, 13-15, 18-24, 29-51, 53-67, 70-71, 83-90, 93-96, 101-105, 108, 120-122, 124-125, 134, 137-174, 176-191, 193-196, 198-200, 202, 208, 211-213, 216-222, 224, 227, 229-239, 243-251, 253-259, 261, 264-280, 282286, 288-289, 291-293, 296-297, 346, 
$348,358-359,370,374,378-379,383$, 387, 395, 399, 401, 403-404, 406-412, 416-428, 432, 439, 458-466, 470-471, 473-474

Vianello, Carlo Antonio, 190, 245, 274, 399

Vico, Giambattista, 432-433, 435, 459

Vignes, Maurice, 122

Villette, sieur de, 135

Vincent de Gournay, Jacques Claude Marie, 70-71, 73-75, 96, 99, 101, 103, 105, 119, 125, 279

Virol, Michèle, 112

Visconti, Alessandro, 399

Visconti, Giuseppe, 257-258, 418

Vitali-Volant, Maria G., 401

Voltaire, François-Marie Arouet de, 71,
108, 264, 291, 409

Wahnbaeck, Till, 293

Walras, Léon, 40-41, 62

Walter, Friedrich, 248

Weinberg, Bennett Alan, 263

White, Allon, 263

Wilczeck, Johann Joseph, 253, 314, 316, 359, 370, 390, 392

Winch, Donald, 452, 474

Windler, Christian, 116

Xifaras, Mikhail, 123

Yntema, Theodore Otte, 62

Zagari, Eugenio, 274, 474

Zamagni, Stefano, 9, 37, 196, 471, 473

Zaninelli, Sergio, 111-112, 114, 398, 400

Zarone, Giuseppe, 400

Ziliotto, Baccio, 339

Zinzendorf, Karl von, 117, 129, 132-134 